\title{
Produção e qualidade de frutos de genótipos de melancia no alto sertão Paraibano, Nordeste Brasileiro ${ }^{1}$
}

\author{
Caciana Cavalcanti Costa ${ }^{2}$, Geraldo Bonifácio da Nóbrega ${ }^{3}$, José Wilson da Silva Barbosa ${ }^{4}$, \\ Lourival Ferreira Cavalcante ${ }^{5}$, Francisco Thiago Coelho Bezerra ${ }^{6}$
}

\begin{abstract}
Resumo: A elevada variabilidade ambiental das áreas cultivadas é um dos fatores preponderantes na produção e qualidade dos produtos agrícolas, sendo essencial à avaliação de genótipos adaptados às diversas situações edafoclimáticas a fim de se obter rendimentos satisfatórios. Diante do exposto, um experimento foi desenvolvido, no período de setembro a novembro de 2014, no município de São Bento, Paraíba, situado na microrregião do Alto Sertão paraibano, com o objetivo de avaliar o comportamento produtivo e a qualidade dos frutos de genótipos de melancieira. Os tratamentos (genótipos de melancieira - BGCIA 863, BGCIA 864, Crimson Sweet e Crimson Select de polpa vermelha, e BGCIA 865, BGCIA 866 de polpa amarela) foram distribuídos em blocos casualizados, com quatro repetições. As variáveis analisadas foram massa média de frutos, produtividades total, comercial e não comercial e as perdas de produção de frutos. Também avaliaram-se os aspectos físicos (diâmetro longitudinal, diâmetro equatorial, índice de formato e espessura da casca) e físico-químicos (teor de sólidos solúveis e acidez titulável na polpa dos frutos). Os genótipos de polpa vermelha, exceto o Crimson Sweet, superam os de polpa amarela na massa dos frutos, produtividade total e comercial e com menores perdas da produção obtida. Para as condições ambientais de São Bento, no alto Sertão do estado da Paraíba, é indicado o cultivo dos genótipos de polpa vermelha (BGCIA 863, BGCIA 864, Crimson Sweet e Crimson Select) em relação aos de polpa amarela (BGCIA 865 e BGCIA 866).
\end{abstract}

Palavras-chave: Citrullus lanatus; Cultivares de melancia; Qualidade de frutos.

\section{Production and quality fruits of watermelon genotypes on backlands Paraibano, Northeast Brazil}

\begin{abstract}
The high environmental variability of cultivated areas is one of the major factors in the production and quality of agricultural products, being essential the evaluation of genotypes adapted to the diverse situations of edaphoclimate in order to obtain satisfactory yields. Thus an experiment was developed during the period September-November 2014, in the municipality of São Bento, Paraiba State, Brazil, located in the micro-region of Alto Sertão paraibano, with the objective to evaluate the of productive behavior and fruit quality genotypes of watermelon. The treatments (genotypes of watermelon BGCIA 863, BGCIA 864, Crimson Sweet e Crimson Selec of red pulp, and BGCIA 865, BGCIA 866 of yellow pulp) were distributed in randomized blocks with four replications. The mean fruit mass, total commercial and non - commercial productivity and fruit production losses were evaluated. Physical aspects were also evaluated (longitudinal diameter, equatorial diameter, shape index and shell thickness) and physical-chemical properties (soluble solids content and titratable acidity in fruit pulp).The genotypes of red pulp, except Crimson Sweet, they outnumber yellow pulp in fruit weight total and commercial productivity and lower production losses obtained. To the environmental conditions of municipality the São Bento, of Alto Sertão of the of Paraíba State, Brazil, should be grown red pulp genotypes (BGCIA 863, 864 BGCIA, Crimson Sweet Crimson and Select) in relation to the yellow pulp (865 BGCIA and BGCIA 866).
\end{abstract}

Keyword: Citrullus lanatus; Watermelon cultivars; Quality of fruits.

\footnotetext{
${ }^{1}$ Submetido em 12/02/2018 e aprovado em 08/04/2018

${ }^{2}$ Doutora em Agronomia; Professora Associada II, Universidade Federal de Campina Grande (UFCG), Centro de Tecnologia Agroalimentar, Unidade Acadêmica de Agronomia e Tecnologia de Alimentos, Pombal-PB, CEP: 58.840-000; E-mail: costacc@ccta.ufcg.edu.br ${ }^{3}$ Engenheiro Agrônomo; Extensionista Rural I, Emater-PB, São Bento-PB, CEP: 58.865-000; E-mail: geraldo_bonifacio@hotmail.com ${ }^{4}$ Engenheiro Agrônomo; Extensionista Rural I, Emater-PB, Pombal-PB, CEP: 58.840-000; E-mail: jwilsonematerpb@hotmail.com ${ }^{5}$ Doutor em Solos e Nutrição de Plantas; Professor Associado IV, Universidade Federal da Paraíba (UFPB), Centro de Ciências Agrária, Programa de Pós-Graduação em Agronomia, Areia-PB, CEP: 58.397-000; E-mail: lofeca@cca.ufpb.br

${ }^{6}$ Doutor em Agronomia; Bolsista PNPD, Universidade Federal da Paraíba (UFPB), Centro de Ciências Agrárias, Programa de Pós-Graduação em Agronomia, Areia-PB, CEP: 58.397-000; E-mail: bezerra_ftc@yahoo.com.br
} 


\section{Introdução}

A melancieira, Citrullus lanatus (Thunb.) Matsum \& Nakai, pertencente à família Curcubitaceae é originária de regiões quentes da África (Filgueira, 2013), sendo atualmente considerada cosmopolita e cultivada em diversos países. Os frutos de melancia possui aceitação mundial, principalmente pelos consumidores dos trópicos, devido ao agradável sabor, propriedades refrescantes e diuréticas associados ao baixo teor calórico. Adicionalmente a isso, o fruto possui propriedades nutricionais e terapêuticas com ação positiva no tratamento de problemas renais, intestinais e respiratórios (Peixoto et al., 2006; Bastos et al., 2008, 2009).

O Brasil é o quarto produtor mundial, ficando atrás da China, Turquia e Irã (FAOSTAT, 2018). Em 2016 a área plantada com melancieiras no Brasil foi de 94.555 hectares, produção de 2.090.432 toneladas e produtividade de 23,11 t $\mathrm{ha}^{-1}$, sendo o cultivo realizado em todas as regiões, mas com destaque para o Nordeste possuindo $31 \%$ da área plantada, $26 \%$ da produção e produtividade de $20,36 \mathrm{t} \mathrm{ha}^{-1}$ (IBGE, 2018). Sendo que o potencial dessa cultura tende a superar aos rendimentos obtidos no Brasil, como constatado por Miranda et al. (2005), 50,7 t ha $^{-1}$; Monção et al. (2012), 36,0 $\mathrm{t} \mathrm{ha}^{-1}$; e por Oliveira et al. (2015), 62,8 t ha ${ }^{-1}$, no Nordeste brasileiro.

Apesar das condições de clima e solo da Paraíba serem adequadas ao cultivo da melancieira, o Estado ainda não é um expressivo produtor; representado em 2016 apenas 1,3\% da área plantada e $0,9 \%$ da produção da Região Nordeste e, com produtividade de 17,89 $\mathrm{t} \mathrm{ha}^{-1}$ (IBGE, 2018). Os seis maiores produtores no Estado da Paraíba são os municípios de Alhandra, São José de Espinharas, Santa Teresinha, Mamanguape, Conde e Pitimbu com 300, 300, $340,420,480$ e 1.200 toneladas, respectivamente (IBGE, 2018). Especificamente no município de São Bento-PB, a melancia é consumida "in natura", estimando-se em 400 toneladas ao ano, contudo, ainda não é explorada comercialmente na região sendo o mercado abastecido pelos produtores potiguares (EMATER-PB, 2008).

$\mathrm{Na}$ literatura há resultados promissores do desempenho produtivo de variedades de melancia quanto ao rendimento e à qualidade para as diferentes regiões do país; essa diversidade de mercado justifica a necessidade de avaliações de materiais genéticos como meta à qualidade que atenda as exigências dos diferentes mercados consumidores brasileiros (Souza et al., 2004; Leão et al., 2006; Ramos et al., 2012).

Pelo exposto, o trabalho foi desenvolvido com o objetivo de avaliar o desempenho produtivo e a qualidade de frutos de genótipos de melancia de frutos vermelhos e amarelos no alto sertão Paraibano.

\section{Material e Métodos}

$\mathrm{O}$ experimento foi conduzido à margem do Rio Piranhas no Sítio Taquarituba, distante quatro $\mathrm{km}$ do município de São Bento, Paraíba. O município de São Bento está situado pelas coordenadas geográficas de Latitude $06^{\circ} 29^{\prime} 10^{\prime \prime}$ S, Longitude $37^{\circ} 27^{\prime} 02^{\prime \prime} \mathrm{W}$ e altitude de $141 \mathrm{~m}$, sob clima Tropical semiárido, distante $450 \mathrm{~km}$ da capital paraibana, João Pessoa, Paraíba.

Os tratamentos constaram da avaliação de seis genótipos de melancieira, quatro de polpa vermelha (BGCIA 863, BGCIA 864, Crimson Sweet e Crimson Select) e dois de polpa amarela (BGCIA 865 e BGCIA 866). Os tratamentos foram distribuídos em blocos ao acaso, com quatro repetições, e a unidade experimental foi formada por oito linhas de seis metros de comprimento. Utilizou-se a densidade de 3.333 plantas por hectare com o arranjo de três metros entre linhas e um metro na linha de cultivo.

As sementes, para a formação das mudas, dos genótipos BGCIA 863, BGCIA 864, BGCIA 865, BGCIA 866 foram cedidas pelo Programa de Melhoramento genético do Centro de Pesquisa Agropecuária do Trópico Semiárido (EMBRAPA Semiárido, Petrolina, PE) e das variedades Crimson Sweet e Crimson Select que foram adquiridas de empresas comerciais, disponíveis no mercado. Os materiais biológicos BGCIA 863 e BGCIA 864 possuem características semelhantes à variedade comercial Crimson Sweet, produzem frutos arredondados, com listas verdes escuras; a variedade Crimson Select produz frutos arredondados, com listas verdeescuras sobre um fundo verde-claro com massa média de 11 a $14 \mathrm{~kg}$ por fruto e polpa vermelha. Os genótipos BGCIA 865 e BGCIA 866 produzem frutos arredondados, com massa de 4 a $6 \mathrm{~kg}$ e polpa amarela. 
Durante a condução do experimento a pluviosidade no local foi de $61 \mathrm{~mm}$, com médias de temperatura e umidade relativa do ar de $28^{\circ} \mathrm{C}$ e $60 \%$, respectivamente. $\mathrm{O}$ solo da área experimental, de acordo com os critérios do Sistema Brasileiro de Classificação de Solos, foi classificado como Luvissolo Crômico Vértico de textura argilosa, com teores de argila, silte e areia de 100,98 e $802 \mathrm{~g} \mathrm{~kg}^{-1}$, respectivamente e os atributos químicos podem ser observados na Tabela 1. Estas análises foram realizadas conforme metodologia compiladas por Teixeira et al. (2011).

Tabela 1 Análise química, na camada de 0-20 cm de profundidade, do Luvissolo Crômico da área experimental em São Bento, Paraíba

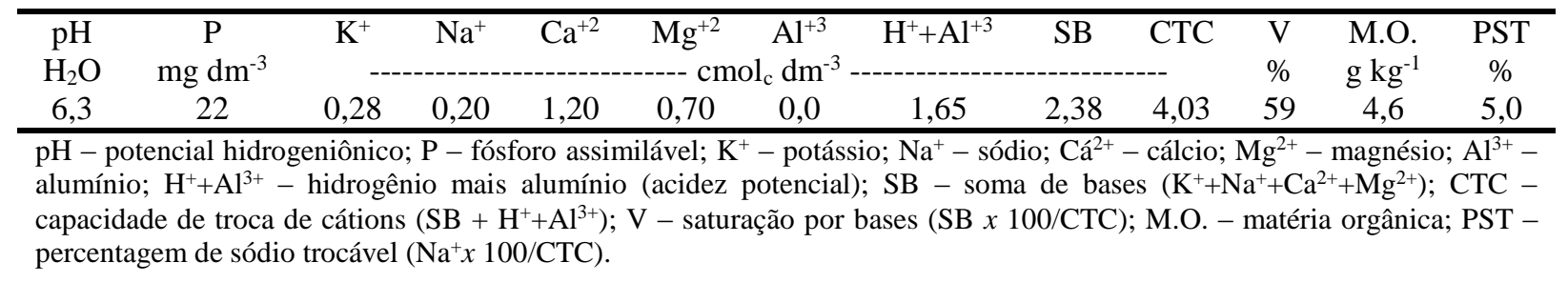

O preparo do solo foi feito em 12/09/2014, com duas gradagens na camada de $0-20 \mathrm{~cm}$, em sentido cruzado. As covas foram abertas nas dimensões de $30 \mathrm{~cm}$ x $30 \mathrm{~cm} \times 30 \mathrm{~cm}$. O manejo da adubação baseou-se nas recomendações de Cavalcanti (2008) para a cultura da melancieira no Estado de Pernambuco. Na adubação de plantio foi incorporado na cova 4,5 $\mathrm{kg}\left(15 \mathrm{tha}^{-1}\right)$ de esterco curtido de gado bovino, $45 \mathrm{~g} \mathrm{(150} \mathrm{kg}$ $\left.\mathrm{ha}^{-1}\right)$ de sulfato de amônio, $36 \mathrm{~g}\left(120 \mathrm{~kg} \mathrm{ha}^{-1}\right)$ superfosfato simples e $10,5 \mathrm{~g}\left(35 \mathrm{~kg} \mathrm{ha}^{-1}\right)$ de cloreto de potássio. As adubações em cobertura constaram de três aplicações de nitrogênio $(25,46$ e 61 dias após o transplantio) e duas de potássio (25 e 46 dias após o transplantio). Na primeira adubação de cobertura aplicou-se 38,5 g por cova de uma mistura de $\mathrm{N}$ (ureia, $45 \% \mathrm{~N})$ e $\mathrm{K}(\mathrm{KCl}$, $60 \% \mathrm{~K}_{2} \mathrm{O}$ ) contendo 155 e $50 \mathrm{~kg} \mathrm{ha}^{-1}$ de ureia e de cloreto de potássio. A segunda com a mesma dose ( $38,5 \mathrm{~g}$ por cova) da mistura com 77,5 $\mathrm{kg} \mathrm{ha}^{-1} \mathrm{de}$ ureia e $50 \mathrm{~kg} \mathrm{ha}^{-1}$ de cloreto de potássio. A última adubação com nitrogênio foi feita aplicando $24 \mathrm{~g}$ por cova de ureia, correspondente a $97 \mathrm{~kg} \mathrm{ha}^{-1}$.

As mudas foram produzidas em bandejas plásticas utilizando o substrato Plantmax ${ }^{\circledR}$, sendo o transplantio realizado quando estavam com quatro folhas definitivas (28/09/2014). A irrigação foi realizada por dois gotejadores de vazão de $4 \mathrm{~L} \mathrm{~h}^{-1}$ em cada planta instalados a uma distância de $20 \mathrm{~cm}$ do caule da planta; sendo a lâmina baseada na evapotranspiração da cultura (ETc), calculada a partir da evapotranspiração de referência (ETo) estimada pelo método de
Penman-Monteith e nos coeficiente de cultura (Kc) da melancieira (Allen et al., 2006).

Os tratos culturais foram capinas manuais com enxada e o controle de pragas e doenças de acordo com a necessidade e aplicações de inseticidas. Nas pulverizações com inseticida utilizou-se imidacloprido contra mosca-branca (Bemisia tabaci) (Agrofit, 2017) e metomil para controle do pulgão verde (Mizus percicae) (Andrei, 2005).

As colheita foram realizadas aos 87 e 91 dias após o transplantio, quando os frutos estavam em maturação comercial (com coloração bem desenvolvida da polpa e teor de sólidos solúveis próximo de $8 \%$ ), colhendo nas 20 plantas centrais da terceira, quarta, quinta e sexta linha de cada parcela. Na colheita foram mensuradas a massa média dos frutos, a produtividade total, comercial e não comercial, e as perdas percentuais de rendimento. Foram considerados frutos comerciais aqueles isentos de rachaduras, danos mecânicos, fitossanitários ou de fundo preto. As perdas foram calculadas entre valores da produtividade não comercial e total.

As avaliações morfológicas dos frutos foram: espessura da casca, medindo-se com paquímetro digital quatro pontos coincidentes da metade superior e inferior do diâmetro e com o ponto de leitura do diâmetro máximo transversal dos frutos; diâmetro médio transversal, medido com régua na região equatorial do fruto; diâmetro longitudinal, medido com régua na região equatorial do fruto; índice de formato do fruto, estimada pela relação entre os valores do 
diâmetro longitudinal e transversal (Diez et al., 2005). A caracterização físico-química constou de sólidos solúveis ( ${ }^{\circ}$ Brix), determinados na polpa dos frutos utilizando refratômetro digital com compensação automática de temperatura $\left(31^{\circ} \mathrm{C}\right)$; acidez titulável (AT), expressa em ácido cítrico, em amostras de $5 \pm 0,01 \mathrm{~g}$ de polpa diluída em $50 \mathrm{~mL}$ de água destilada, titulado com $\mathrm{NaOH}$ $0,1 \mathrm{~N}$ (Zenebon et al., 2008).

Os dados foram submetidos à análise de variância e as médias comparadas pelo teste de Tukey $(\mathrm{p} \leq 0,05)$.

\section{Resultados e Discussão}

Os cultivares que produziram frutos de maior massa média foram BGCIA 863, Crimson Select e BGCIA 864, enquanto os genótipos BGCIA 865 , Crimson Sweet e BGCIA 866 foram os que produziram os frutos com menor massa (Tabela 2). A massa média dos frutos das cultivares Crimson Sweet e Crimson Select ficaram abaixo dos seus receptivos potenciais de 11-14 e 12-15 $\mathrm{kg}$ (Feltrin, 2017). Carmo et al. (2015), analisando genótipos de melancia em Latossolo Amarelo no município de Boa Vista-RR, obtiveram frutos de Crimson Sweet com massa média de 7,8 kg. Enquanto no semiárido brasileiro foram encontradas médias da massa do fruto de Crimson Sweet de 6,2 $\mathrm{kg}$, por Oliveira et al. (2015) avaliando épocas de plantio em um Argissolo Vermelho Amarelo no município de Mossoró-RN, e entre 6,9 a 8,8 kg, por Nascimento et al. (2017) estudando doses de esterco em Neossolo Flúvico no município de Patos-PB. Estando esse caractere fortemente influenciado pelas condições edafoclimáticas.

Tabela 2 Massa média de fruto (MMF), produtividade total (PT), comercial (PC) e não comercial (PNC), e perdas da produção (PP) de genótipos de melancia no município de São Bento, sertão Paraibano

\begin{tabular}{|c|c|c|c|c|c|}
\hline \multirow{2}{*}{ Genótipos } & MMF & $\mathrm{PT}$ & $\mathrm{PC}$ & PNC & \multirow{2}{*}{$\begin{array}{c}\mathrm{PP} \\
\%\end{array}$} \\
\hline & $\mathrm{kg}$ por fruto & ------------ & por hectare --- & -------- & \\
\hline BGCIA 863 & $8,22 \mathrm{a}$ & $27.411,50 \mathrm{a}$ & $25.811,75 \mathrm{a}$ & $1.599,75 \mathrm{a}$ & $\overline{5,83}$ \\
\hline BGCIA 864 & $6,51 \mathrm{abc}$ & $21.707,25 \mathrm{abc}$ & $21.374,00 \mathrm{abc}$ & $333,25 \mathrm{~b}$ & 1,53 \\
\hline BGCIA 865 & $5,05 \mathrm{~cd}$ & $16.862,00 \mathrm{bcd}$ & $15.554,00 \mathrm{~cd}$ & $1.308,00 \mathrm{a}$ & 7,75 \\
\hline BGCIA 866 & $4,20 \mathrm{~d}$ & $14.007,25 \mathrm{~d}$ & $12.624,25 \mathrm{~d}$ & $1.383,00 \mathrm{a}$ & 9,87 \\
\hline Crimson Sweet & 5,86 bcd & $19.555,00 \mathrm{bc}$ & $18.753,24 \mathrm{bc}$ & $801,76 \mathrm{~b}$ & 4,10 \\
\hline Crimson Select & $7,18 \mathrm{ab}$ & $23.934,75 \mathrm{ab}$ & $22.446,50 \mathrm{ab}$ & $1.488,25 \mathrm{a}$ & 6,21 \\
\hline $\mathrm{DMS}^{1}$ & 1,82 & $6.098,81$ & $6.038,13$ & $4.266,88$ & - \\
\hline $\mathrm{CV}(\%)$ & 12,89 & 12,90 & 13,44 & 179,43 & - \\
\hline
\end{tabular}

${ }^{1} \mathrm{DMS}$ - diferença mínima significativa;

Médias seguidas pela mesma letra, na coluna, não diferem estatisticamente pelo Teste de Tukey $(\mathrm{p} \leq 0,05)$.

As menores produtividades, total e comercial, foram produzidas pelos genótipos de polpa amarela BGCIA 865 e BGCIA 866, enquanto as maiores foram registadas para os genótipos de polpa vermelha BGCIA 863, BGCIA 864 e Crimson Select (Tabela 2). Essas inferioridades, em geral, são respostas das diferenças de ambientes, entre o local que foi produzido os materiais genéticos e, aquele a que foram submetidos para crescimento e produção; apesar da semelhança, em termos de luminosidade, temperatura e umidade relativa do ar, entre os ambientes de Petrolina, PE e São Bento, PB. Dentre os genótipos, o BGCIA 866 foi o menos eficiente com a menor produção total e maior perda de produção. No entanto, este genótipo apresenta potencial para satisfazer os consumidores que preferem frutos de tamanho médio ou das minimelancias, os quais criaram um nicho de mercado novo de frutos e hortaliças, determinado pela mudança no comportamento das pessoas, que vivem nos grandes centros urbanos, os quais moram em espaços pequenos e com famílias menores.

O Nordeste brasileiro concentra a maior produção de melancia, representando em 2016 $26 \%$ da produção nacional, mas em termos de produtividade registra-se nessa região o menor valor que, em média, foi de $21 \mathrm{tha}^{-1}$ sendo a maior no Centro-Oeste com $34 \mathrm{t} \mathrm{ha}^{-1}$ (IBGE, 2018). Porém, o potencial dessa cultura supera os registrados nesse trabalho e a média da região, com produtividades de até 50,7 $\mathrm{t} \mathrm{ha}^{-1}$, em Neossolo Quartzarênico no município de Paraipaba-CE (Miranda et al., 2005), de 36,0 tha' ${ }^{1}$, em Latossolo Amarelo no município de Santa 
Rita de Cássia-BA (Monção et al., 2012), e de $62,8 \mathrm{t} \mathrm{ha}^{-1}$, em Argissolo Vermelho Amarelo no município de Mossoró-RN (Oliveira et al., 2015).

Ao relacionar os valores da produção não comercial (PNC) com a produção total, constatam-se que as maiores perdas de 9,87 e $7,75 \%$ foram registradas nas colheitas dos frutos dos genótipos de polpa amarela BGCIA 866 e BGCIA 865, respectivamente (Tabela 2). As menores perdas de rendimento referiram-se aos genótipos BGCIA 864 (1,53\%) e Crimson Sweet $(4,10 \%)$, sendo este último o mais cultivado no país, tanto pela aceitabilidade de mercado como pela sua adaptabilidade em todas as regiões brasileiras (Leão et al., 2008).

Os genótipos BGCIA 863, BGCIA 864, Crimson Sweet e Crimson Select, de polpa vermelha, não divergiram em relação às variáveis espessura da casca e diâmetros longitudinal e transversal (Tabela 3). Os frutos com maiores dimensões estão relacionados a sua maior massa média e que, provavelmente, após estimulam espessamento da casca. Os genótipos BGCIA 865 e BGCIA 866, de polpa amarela, produziram frutos com menor espessura de casca, diâmetro longitudinal e diâmetro transversal. Essa situação evidencia a necessidade de se avaliar as plantas quanto aos aspectos nutricionais em relação aos nutrientes que promovem a firmeza da casca dos frutos como cálcio ou ao melhoramento genético destes genótipos quanto a esta característica. Ambas as situações, contribuem para a proteção do fruto às intempéries no campo, bem como, maior vida útil e melhor resistência de empilhamento, armazenamento e transporte (Barros et al., 2012).

Tabela 3 Espessura da casca (EC), diâmetro longitudinal (DL), diâmetro equatorial (DE), índice de formato do fruto (IFF), teor de sólidos solúveis (SS) e acidez titulável (AT) em frutos de genótipos de melancia

\begin{tabular}{|c|c|c|c|c|c|c|}
\hline \multirow{2}{*}{ Genótipos } & $\mathrm{EC}$ & $\mathrm{DL}$ & DT & IFF & $\mathrm{SS}$ & AT \\
\hline & \multicolumn{3}{|c|}{---------------- cm ---------------- } & DL:DE & $\%$ & $\mathrm{mg} 100^{-1} \mathrm{~g}$ \\
\hline BGCIA 863 & $1,88 \mathrm{a}$ & $27,16 \mathrm{a}$ & $24,02 \mathrm{a}$ & $1,13 \mathrm{a}$ & $10,24 \mathrm{a}$ & $0,096 \mathrm{~b}$ \\
\hline BGCIA 864 & $2,06 \mathrm{a}$ & $26,65 \mathrm{a}$ & $22,93 \mathrm{ab}$ & $1,16 \mathrm{a}$ & $9,53 \mathrm{ab}$ & $0,120 \mathrm{~b}$ \\
\hline BGCIA 865 & $0,86 \mathrm{~b}$ & $22,63 \mathrm{~b}$ & $20,19 \mathrm{bc}$ & $1,12 \mathrm{a}$ & $8,35 \mathrm{~b}$ & $0,865 \mathrm{a}$ \\
\hline BGCIA 866 & $0,82 \mathrm{~b}$ & $20,61 \mathrm{~b}$ & $19,15 \mathrm{c}$ & $1,07 \mathrm{a}$ & $8,37 \mathrm{~b}$ & $0,073 \mathrm{~b}$ \\
\hline Crimson Sweet & $2,04 \mathrm{a}$ & $25,64 \mathrm{a}$ & $22,23 \mathrm{abc}$ & $1,15 \mathrm{a}$ & $9,57 \mathrm{ab}$ & $0,100 \mathrm{~b}$ \\
\hline Crimson Select & $2,08 \mathrm{a}$ & $27,59 \mathrm{a}$ & $23,62 \mathrm{a}$ & $1,16 \mathrm{a}$ & $9,85 \mathrm{ab}$ & $0,086 \mathrm{~b}$ \\
\hline $\mathrm{DMS}^{1}$ & 0,69 & 2,90 & 3,35 & 0,16 & 1,61 & 0,186 \\
\hline $\mathrm{CV}(\%)$ & 18,60 & 5,03 & 6,61 & 6,28 & 20,82 & 8,66 \\
\hline
\end{tabular}

${ }^{1} \mathrm{DMS}$ - diferença mínima significativa;

Médias seguidas pela mesma letra nas colunas não diferem estatisticamente pelo Teste de Tukey $(\mathrm{p} \leq 0,05)$.

Quanto aos índices de formato de frutos verificam-se valores próximos de 1 e sem diferença significativa entre os frutos dos genótipos (Tabela 3). Essa situação, indica a produção de frutos com formato redondo, conforme sugere o programa brasileiro para a melhoria dos padrões comerciais e embalagem dos hortifrutigranjeiros (CEAGESP, 2000). Os resultados dos genótipos BGCIA 863 e BGCIA 864 em relação aos tradicionalmente cultivados Crimson Sweet e Crimson Select são satisfatórios, uma vez que a manutenção do formato do fruto próximo da unidade é uma das características de exigências do mercado consumidor.

Assim como para a espessura da casca, diâmetro longitudinal e transversal, os teores de sólidos solúveis em frutos dos genótipos de polpa vermelha BGCIA 863, BGCIA864, Crimson Sweet e Crimson Select não variaram entre si, mas, em geral, superaram os valores dos frutos de polpa amarela, BGCIA 865 e BGCIA 866 (Tabela $3)$. Nos frutos de polpa vermelha os sólidos solúveis variaram de 9,53 a $10,24 \%$ entre os genótipos BGCIA864 e BGCIA 863 e de 9,57 a 9,85\% entre Crimson Sweet e Crimson Select, respectivamente. Comparativamente, esses valores superam os $6,86 \%$ obtidos por Leão et al. (2006) ao avaliarem oito genótipos de melancieira em Águas Limpas, Distrito Federal. Frutos de melancia de polpa vermelha devem possuir no mínimo teor de sólidos solúveis de $9^{\circ}$ Brix, conforme recomendação da União Europeia, devendo-se, entretanto, preferir valores 
a partir de $10^{\circ}$ Brix, que são mais bem aceitos pelo mercado interno (Dias e Lima, 2010).

Os genótipos de polpa vermelha (BGCIA 863, BGCIA 864, Crimson Sweet e Crimson Select) produziram frutos com menor acidez que o de polpa amarela, BGCIA 865. Ramos et al. (2012) avaliando espaçamentos combinados com genótipos de melancieiras diploides, sendo oito híbridos experimentais, provenientes do Programa de Melhoramento Vegetal da Embrapa Semiárido e duas cultivares comerciais (Sugar Baby e Smile), constataram que a acidez titulável variou de 0,08 a 0,14 (mg ácido cítrico $100^{-1} \mathrm{~g}$ ).

\section{Conclusão}

Os componentes da produção e da qualidade dos frutos dos genótipos de polpa vermelha BGCIA 863 e 864 foram semelhantes aos dos genótipos Crimson Sweet e Crimson Select tradicionalmente cultivados;

Os genótipos de polpa amarela BGCIA 865 e BGCIA 866 são menos produtivos, as perdas das colheitas são maiores e os frutos são de qualidade inferior aos dos genótipos de polpa vermelha;

Para as condições ambientais de São Bento, no alto Sertão do estado da Paraíba, é indicado a cultivo dos genótipos de polpa vermelha (BGCIA 863, BGCIA 864, Crimson Sweet e Crimson Select) em relação aos de polpa amarela (BGCIA 865, BGCIA 866).

\section{Referências}

AGROFIT (Sistema de Agrotóxicos Fitossanitários). Disponível em: < http://agrofit.agricultura.gov.br/agrofit_cons/principal_ag rofit_cons > Acesso: 10 de Outubro de 2017.

Allen, R. G.; Pereira, L. S.; Raes, D.; Smith, M. Evapotranspiración del cultivo: guías para la determinación de los requerimentos de agua de los cultivos. Itália: FAO (Estúdio FAO: Riego y Drenaje, 56), 2006. 298p. http://www.fao.org/3/ax0490s.pdf

Andrei, E. 2005 Compêndio de defensivos agrícolas: guia prático de produtos fitossanitários para uso agrícola. $7^{\mathrm{a}}$ ed. São Paulo: Organização Andrei, 2005. 1141 p.

Barros, M. M.; Araújo, W. F.; Neves, L. T. B. C.; Campos, A. J de; Tosin, J. M. Produção e qualidade da melancia submetida a adubação nitrogenada. Revista Brasileira de Engenharia Agrícola e Ambiental, v.16, n.10, p.1078-1084, 2012. http://dx.doi.org/10.1590/S1415$\underline{43662012001000007}$

Bastos F. G. C.; Azevedo, B. M. de; Rego, J. de L.; Viana, T. V. de A.; D’Ávila, H. T. Efeitos de espaçamentos entre plantas na cultura da melancia na Chapada do Apodi, Ceará. Revista Ciência Agronômica, v.39, n.2, p.240-244, 2008.

http://ccarevista.ufc.br/seer/index.php/ccarevista/article/vi ew/54

Carmo, I. L. G. da S.; Silva, E. S.; Monteiro Neto, J. L. L.; Trassato, L. B.; Medeiros, R. D. de; Porto, D. S. Desempenho agronômico de cultivares de melancia no cerrado de Boa Vista, Roraima. Revista Agro@mbiente, v.9, n.3, p.268-274, 2015. http://dx.doi.org/10.18227/19828470ragro.v9i3.2531

Cavalcanti, F. J. de A. (Ed.). Recomendações de adubação para o estado de Pernambuco: $2^{\text {a }}$ aproximação. 3 ed. Recife: Instituto Agronômico de Pernambuco, 2008. 212p.

CEAGESP (Companhia de Entrepostos e Armazéns Gerais de São Paulo). Programa Padrão: programa brasileiro para a melhoria dos padrões comerciais e embalagem dos hortigranjeiros. São Paulo: CEAGESP, 2000. $564 \mathrm{p}$.

Dias, R. C. S.; Lima, M. A. C. Sistema de Produção de Melancia. Embrapa Semiárido: Petrolina. 2010. Disponível em < http://sistemasdeproducao.cnptia.embrapa.br/FontesHTM L/Melancia/SistemaProducaoMelancia/index.htm >. Acesso em 18 de mar de 2013 (Sistema de produção 6, Versão eletrônica).

Diez, M. J.; Dooijeweert, W.; Maggioni, L.; Lipman, E. 2005. Appendix III. Minimum descriptor lists for Cucurbita, cucumber, melon and watermelon. In: REPORT OF A WORKING GROUP ON CUCURBITS, 1, 2005 Bulgaria. Report...Bulgaria: IPGRI. P. 21-32. Disponível em: < http://www.ecpgr.cgiar.org/fileadmin/templates/ecpgr.org lupload/NW_and_WG_UPLOADS/Cucurbits_Descriptor Lists.pdf >. Acesso em: 7 nov. 2009. Documentos (INFOTECA-E)

Teixeira, P. C.; Donagema, G. K.; Fontana, A.; Texeira, W. G. M. (Eds.). Manual de Métodos de Análise de Solo. $3^{\mathrm{a}}$ ed. Brasília: Embrapa, 2017. 
http://ainfo.cnptia.embrapa.br/digital/bitstream/item/1719 07/1/Manual-de-Metodos-de-Analise-de-Solo-2017.pdf

EMATER-PB (Empresa de Assistência Técnica e Extensão Rural da Paraíba). Unidade Operativa de São Bento-PB: Levantamento sobre o consumo de melancia no Município de São Bento - PB em Julho de 2008. (Relatório de atividades), 2008. 34p.

FAOSTAT. Crops. Disponível em: < http://fao.org/faostat/en/\#data/QC >. Acessoem: 28 jan. 2017.

Feltrin. Catálogo: winners e commodities. 2 ed. Feltrin ${ }^{\circledR}$ sementes, 2017.61p.

Ferreira, G. N.; Suguino, E.; Martins, A. N.; Campagnol, R.; Furlaneto, F. de P. B.; Minami, $K$. A cultura da melancia. Piracicaba: ESALQ, 2013. 62p. (Série Produtor Rural, ${ }^{\circ}$ 54).

Filgueira, F. A. R. Novo Manual de Olericultura: agrotecnologia moderna na produção e comercialização de hortaliças. $3^{\mathrm{a}} \mathrm{ed}$. Viçosa: Editora UFV, 2012. 421p.

IBGE (Instituto Brasileiro de Geografia e Estatística). Produção Agrícola Municipal. Disponível em: < https://sidra.ibge.gov.br/tabela/1612 > Acesso: 10 de janeiro de 2018.

Leão, D. S.; Peixoto J. R.; Vieira J. V. Cecílio Filho, A. B. Produtividade de melancia em diferentes níveis de adubação química e orgânica. Bioscience Journal, v.24, n.4, p.3241 , 2008. http://seer.ufu.br/index.php/biosciencejournal/article/view $\underline{16630}$

Leão, D. S.; Peixoto J. R.; Vieira J. V. Teor de licopeno e de sólidos solúveis totais em oito cultivares de melancia. Bioscience Journal, v.22, n.3, p.7-15, 2006. http://seer.ufu.br/index.php/biosciencejournal/article/view 16629

Monção, O. P.; Ribeiro, J. J.; Moscon, S. E.; Oliveira, D. N. S. de; Nascimento Neto, J. G. Produtividade da cultura da melancia sob diferentes doses de potássio no município de Santa Rita de Cássia-BA. Enciclopédia Bioesfera, v.8, n.15, p.1423-1431, 2012. http://conhecer.org.br/enciclop/2012b/ciencias\%20agraria s/produtividade\%20da\%20cultura.pdf

Nascimento, J. A. M. do; Souto, J. S.; Cavalcante, L. F.; Medeiros. S. A da S.; Pereira, W. E. Produção de melancia em solo adubado com esterco bovino e potássio. Revista Brasileira de Ciências Agrárias, v.12, n.2, p.122-127, 2017. http://doi:10.5039/agraria.v12i2a5427

Oliveira, J. B. de; Grangeiro, L. C.; Sobrinho, J. E.; Moura, M. S. B. de; Carvalho, C. A. C. de. Rendimento e qualidade de frutos de melancia em diferentes épocas de plantio. Revista Caatinga, v.28, n.2, p.19-25, 2015. http://periodicos.ufersa.edu.br/index.php/caatinga/article/ view/3324

Peixoto J. R.; Leão D. S.; VieiraJ. V. Teor de licopeno e de sólidos solúveis totais em oito cultivares de melancia. Bioscience Journal, v. 22, n.3, p. 7-15, 2006. http://www.seer.ufu.br/index.php/biosciencejournal/articl e/view/6629/4357

Ramos, A. R. P.; Dias, R. C. S.; Aragão, C. A.; Batista, P. F; Pire, M. M. da L. Desempenho de genótipos de melancia de frutos pequenos em diversas densidades de plantio. Horticultura Brasileira, v.30, n.2, p.333-338, 2012. http://www.scielo.br/pdf/hb/v30n2/v30n2a25.pdf

Souza, F. F.; Gama, F. C.; Queiróz, M. A. Análise da capacidade de combinação em cruzamentos dialélicos de três genótipos de melancia. Horticultura Brasileira, v.22, n.4, p.789-793, $2004 . \quad$ http://dx.doi.org/10.1590/S0102$\underline{05362004000400025}$

Zenebon, O.; Pascuet, N. S.; Tiglea, P. (Eds.). Métodos físico-químicos para análise de alimentos. $4^{\mathrm{a}}$ ed. São Paulo: Instituto Adolfo Lutz, 2008.1020p. http://www.ial.sp.gov.br/resources/editorinplace/ial/2016 _3_19/analisedealimentosial_2008.pdf 